\title{
MACUSTAR: Development and Clinical Validation of Functional, Structural, and Patient-Reported Endpoints in Intermediate Age-Related Macular Degeneration
}

\author{
Robert P. Finger ${ }^{\text {a }}$ Steffen Schmitz-Valckenberg ${ }^{\text {a }}$ Matthias Schmid ${ }^{\text {b }}$ \\ Gary S. Rubin ${ }^{c}$ Hannah Dunbarc Adnan Tufaild David P. Crabbe \\ Alison Binns ${ }^{e}$ Clara I. Sánchez ${ }^{f}$ Philippe Margaron ${ }^{g} \quad$ Guillaume Normand $^{g}$ \\ Mary K. Durbin ${ }^{h} \quad$ Ulrich F.O. Luhmann ${ }^{i}$ Parisa Zamirig José Cunha-Vaz ${ }^{j}$ \\ Friedrich Asmus $^{k}$ Frank G. Holz ${ }^{a}$ on behalf of the MACUSTAR consortium

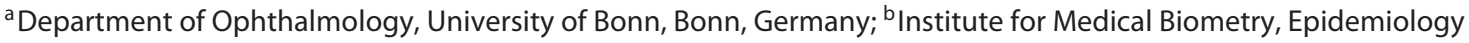
and Informatics, University Hospital Bonn, Bonn, Germany; ' UCL Institute of Ophthalmology, University College London, London, UK; ${ }^{d}$ Moorfields Eye Hospital, London, UK; ${ }^{e}$ Division of Optometry and Visual Science, School of Health Sciences, City, University of London, London, UK; ${ }^{f}$ Diagnostic Image Analysis Group, Radboud University

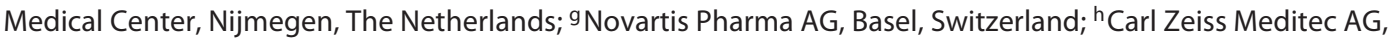
Dublin, CA, USA; 'Roche Pharmaceutical Research and Early Development, Translational Medicine Ophthalmology, Roche Pharma Research and Early Development, Roche Innovation Center Basel, Basel, Switzerland; ' Association for Innovation and Biomedical Research on Light and Image, Coimbra, Portugal; ${ }^{k}$ Development Pharmaceuticals,
\end{abstract} \\ Therapeutic Areas PAO, Bayer AG, Berlin, Germany
}

\section{Keywords}

Intermediate age-related macular degeneration .

Disease progression $\cdot$ Clinical endpoint $\cdot$ Patient-reported outcomes $\cdot$ Structure-function correlation

\begin{abstract}
Purpose: Currently, no outcome measures are clinically validated and accepted as clinical endpoints by regulatory agencies for drug development in intermediate age-related macular degeneration (iAMD). The MACUSTAR Consortium, a public-private research group funded by the European Innovative Medicines Initiative intends to close this gap. Procedures: Development of study protocol and statistical anal-
\end{abstract}

ysis plan including predictive modelling of multimodal endpoints based on a review of the literature and expert consensus. Results: This observational study consists of a cross-sectional and a longitudinal part. Functional outcome measures assessed under low contrast and low luminance have the potential to detect progression of visual deficit within iAMD and to late AMD. Structural outcome measures will be multimodal and investigate topographical relationships with function. Current patient-reported outcome mea-

MACUSTAR Consortium Members: M. Berger, J. Bohner, M. Böttger, J.E. Brazier, T. Butt, C. Carapezzi, J. Carlton, M. Costa, A. Ferrao, C. Hoyng, F. Kersten, J. Krätzschmar, A. Lüning, C. Martinho, D. Rowen, J. Sahel, D. Sanches Fernandes, A. Skelly, C. Wojek.

\begin{tabular}{ll}
\hline KARGER & $\begin{array}{l}\text { Karger } \\
\text { Published by S. Karger AG, Basel }\end{array}$ \\
E-Mail karger@karger.com & This article is licensed under the Creative Commons Attribution- \\
www.karger.com/oph & $\begin{array}{l}\text { NonCommercial-NoDerivatives 4.0 International License (CC BY- } \\
\text { NC-ND) (http://www.karger.com/Services/OpenAccessLicense). } \\
\text { Usage and distribution for commercial purposes as well as any dis- } \\
\text { tribution of modified material requires written permission. }\end{array}$
\end{tabular}


sures (PROMs) are not acceptable to regulators and may not capture the functional deficit specific to iAMD with needed precision, justifying development of novel PROMs for iAMD. The total sample size will be $n=750$, consisting mainly of subjects with iAMD $(n=600)$. Conclusions: As clinical endpoints currently accepted by regulators cannot detect functional loss or patient-relevant impact in iAMD, we will clinically validate novel candidate endpoints for iAMD.

\section{C) 2018 The Author(s)}

Published by S. Karger AG, Basel

\section{Introduction}

Age-related macular degeneration (AMD) affects almost $30 \%$ of the older population and progresses slowly from early AMD to intermediate AMD (iAMD) and ultimately late-stage AMD with severe and frequently irreversible visual loss over a decade [1]. Population aging will lead to a considerable increase in AMD prevalence. Today, late-stage AMD is the leading cause of blindness among the elderly in industrialized countries and affects more than 2.5 million patients in the EU [2-4] resulting in direct annual costs of over 2 billion Euros [5].

In order to reduce the significant burden of late AMD, novel interventions that stop or delay progression from iAMD to late AMD will need to be developed [6]. This has been highlighted by the number of failed studies to prevent the progression of geographic atrophy (GA) with different approaches such as inhibiting complement and visual cycle modulators. Parallels with Alzheimer's disease have been noted, where it is felt that part of the failure of clinical trials (CTs) to date has been the onset of treatment too late in the disease process $[7,8]$. For CT clinical endpoints validated and accepted by regulatory agencies, health technology assessment (HTA) bodies, and payers are needed. Currently, these do not exist for iAMD CTs. The currently accepted endpoints like high-contrast, high-luminance best-corrected visual acuity (BCVA), are not appropriately reflecting the visual deficit in iAMD or only after progression to late $A M D$, which may require an observation period of up to several years. Therefore, BCVA is not an adequate endpoint for iAMD CTs.

Still, there is good evidence indicating that patients with iAMD experience impairment of visual function, yet it is unknown to what extent this impacts the patients' life or how it can reliably be measured and quantified [9-11]. It is also not well known which the specific risk factors in the population of iAMD patients are to identify those with more rapid progression to late stages of the disease.
Therefore, the successful development of iAMD interventions requires validated functional, morphological, and patient-reported endpoints for CTs which are clinically meaningful and accepted by regulatory agencies. In addition, functional decline in iAMD and specific risk factors for iAMD progression to late AMD need to be better characterized to inform and improve conduct of future iAMD CTs.

Against this background, the major objective of the MACUSTAR consortium is to develop novel clinical endpoints for CTs with a regulatory and patient access intention in the area of functional, structural, and patient-reported outcome measures in patients with iAMD.

For this objective, it is not sufficient to test different endpoint categories in isolation, e.g. optimizing an imaging parameter without providing functional data to demonstrate that progression of disease shown by images is also reflected in functional measures and/or measures of patient-reported health. Specifically, this holistic and integrative approach for prospectively looking for correlation of candidate endpoints in one validation study is one of the unique strengths of the MACUSTAR project.

Using this approach, MACUSTAR will also characterize visual impairment in iAMD and its progression, as well as identify risk factors for progression to late-stage AMD. Herein, we report the process of selection and optimization of candidate outcome measures, protocol development, and considerations for statistical analyses for the MACUSTAR clinical study which will deliver the above-mentioned clinical endpoints.

\section{Materials and Methods}

\section{Consortium Structure}

The MACUSTAR consortium (online suppl. material; for all online suppl. material, see www.karger.com/doi/10.1159/491402) was awarded funding by the European Union Innovative Medicines Initiative 2 (IMI2) based on the Call 7 Topic 4 (No. IMI22015-07-04): "Dry Age-Related Macular Degeneration: Development of Novel Clinical Endpoints for Clinical Trials with a Regulatory and Patient Access Intention."

The call topic text used the term "dry" AMD, which only separates AMD with choroidal neovascularization (CNV) (wet AMD) form all other AMD forms (dry AMD). In the MACUSTAR project (and as also suggested in the call topic text), the Beckman classification of AMD will be used as this much better reflects the progressive nature of AMD [12]. In line with the description of the call topic text, the MACUSTAR project will focus on assessment of patients with iAMD and the progression of iAMD to late-stage AMD (comprising neovascular wet AMD and atrophic AMD or GA). Patients with no AMD, early AMD, and neovascular AMD will be included in cross-sectional assessments to demonstrate the
62

Ophthalmologica 2019;241:61-72

DOI: $10.1159 / 000491402$
Finger et al. 


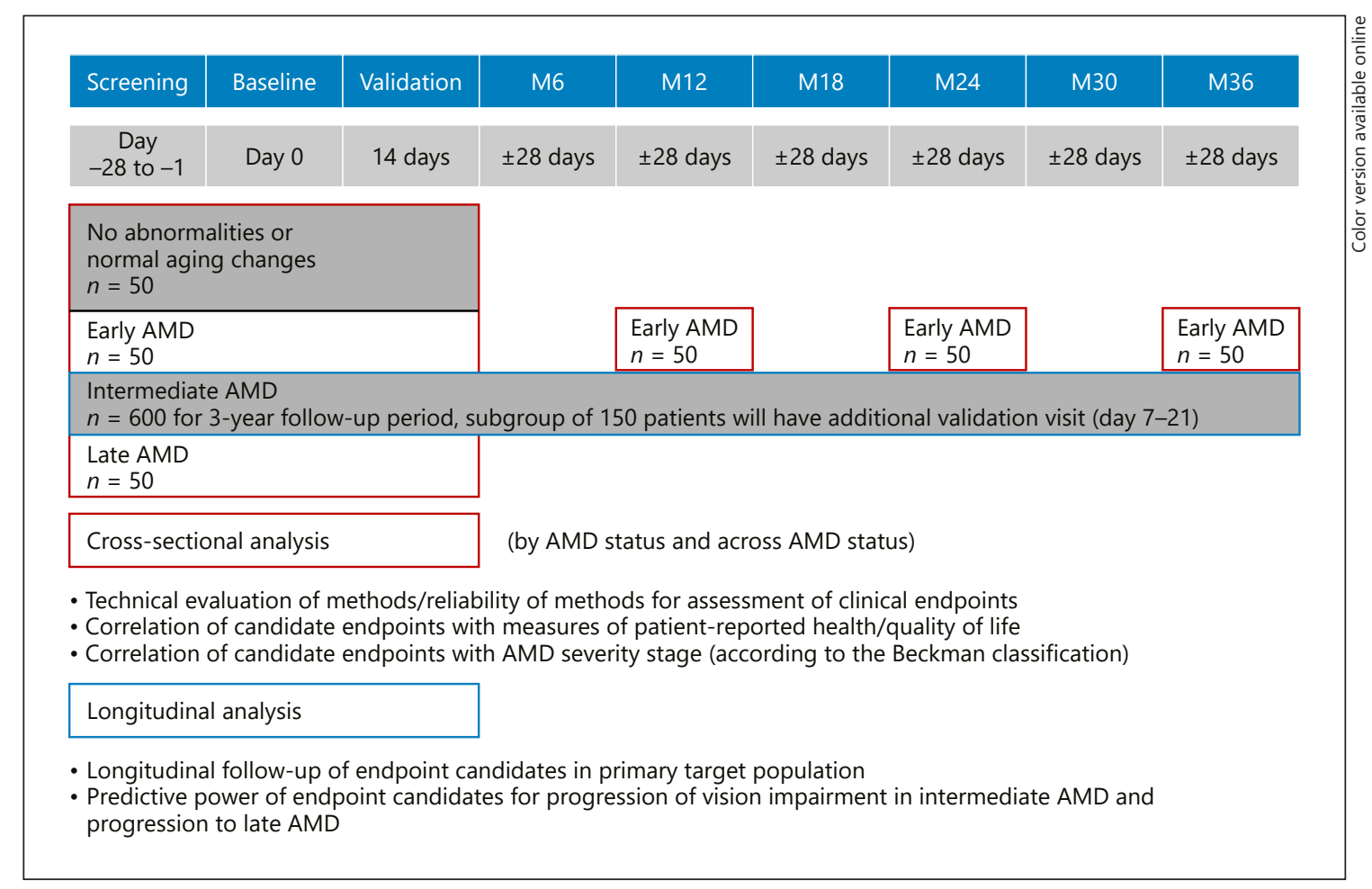

Fig. 1. Overall structure of the MACUSTAR clinical study.

degree of discrimination of iAMD and its progression to late-stage AMD by the candidate endpoints.

IMI2 is the world's largest public private partnership in life sciences, with the EU providing funding which is matched by the European Federation of Pharmaceutical Industries and Associations (EFPIA) and all projects include academic and industrial partners (EFPIA members and associated companies). Researchers from academia and industry work actively and jointly on all parts of research project. Decision bodies of IMI2 consortia have a 1:1 distribution of academic and industry votes.

To optimally address the research question, the MACUSTAR consortium is structured in five work packages (WPs; see online suppl. material). These include conduct of the clinical study (WP1 - Clinical Trial), functional outcome measures (WP2 Functional Endpoints), patient-reported outcome measures (WP3 - Patient-reported Heath and Health economics), structural outcome measure/imaging (WP4 - Anatomical and Imaging Endpoints), and project coordination (WP5 - Project Management, Communication, and Dissemination).

\section{Protocol Development}

Based on a review of the available literature up to September 2016 on PubMed as well as expert consensus within the consortium (see list of members) and with regulators, candidate outcome measures for clinical validation as endpoints were identified. Outcome measures were chosen to be included into the MACUSTAR clinical study if there were data supporting their use in iAMD, sufficient evidence to support adequate quality of the measurement obtained, testing could be standardized across

MACUSTAR - Clinical Validation of iAMD Endpoints multiple sites, and test burden would be compatible with a multisite setting and repetition of measurements for a follow-up of typically $2-3$ years.

Study design was chosen to allow for the most comprehensive evaluation of chosen outcome measures, including assessments of reliability, responsiveness to change over time, structure-function correlation, and patient relevance.

\section{Interaction with Regulators and HTA Bodies}

The MACUSTAR consortium targets validation and acceptance of candidate endpoints by regulatory and HTA bodies. As such, it is central to early on align the validation strategy. Specifically, the design and analysis approach for clinical data acquisition and generation are developed with external input by stakeholders. For the MACUSTAR clinical study, feedback was implemented from patient organizations such as Retina International. In addition, the consortium initiated an official scientific advice procedure on biomarker qualification from regulatory agencies and HTA bodies early on in 2016/2017 before the active funding phase of the project. The scientific advice procedure including EMA, US FDA, and NICE was completed in April 2017 with a letter of support to the project issued by EMA (www.ema.europa.eu).

Discussions and feedback on general approaches for biomarker qualification in iAMD, on how to best correlate the different endpoint categories, and on the clinical study design and statistical analyses were integrated into the subsequent protocol development, sample size calculations, and project planning.

Two further follow-on joint scientific advices with EMA, US FDA, and NICE are targeted for biomarker qualification with (1) 
Table 1. Definitions of AMD disease stages used in MACUSTAR

\begin{tabular}{llll}
\hline $\begin{array}{l}\text { Subjects } \\
\text { planned }\end{array}$ & AMD category & $\begin{array}{l}\text { Morphological criteria according to the } \\
\text { Beckmann classification }\end{array}$ & As applied in the MACUSTAR clinical study \\
\hline 50 & $\begin{array}{l}\text { No apparent aging } \\
\text { changes (no AMD) } \\
\text { Normal aging } \\
\text { changes (no AMD) }\end{array}$ & $\begin{array}{l}\text { No drusen and no AMD pigmentary } \\
\text { abnormalities } \\
\text { Only drupelets (small drusen } \leq 63 \mu \mathrm{m} \text { ) and } \\
\text { no AMD pigmentary abnormalities* }\end{array}$ & $\begin{array}{l}\text { No or normal aging changes (in both eyes) are } \\
\text { the same category in the MACUSTAR study, } \\
\text { age-matched }\end{array}$ \\
\hline 50 & $\begin{array}{l}\text { Early AMD (both } \\
\text { eyes) }\end{array}$ & $\begin{array}{l}\text { Medium drusen }>63 \text { and } \leq 125 \mu \mathrm{m}_{\text {and }} \\
\text { no AMD pigmentary abnormalities }\end{array}$ & Bilateral early AMD, i.e. both eyes \\
\hline 600 & $\begin{array}{l}\text { Intermediate AMD } \\
\text { (iAMD) }\end{array}$ & $\begin{array}{l}\text { Large drusen }>125 \mu \mathrm{m} \text { and/or } \\
\text { any AMD pigmentary abnormalities }\end{array}$ & $\begin{array}{l}\text { a } \\
\text { Bilateral iAMD or iAMD in the study eye and } \\
\text { size } 1.25 \text { mm }{ }^{2} \text { ) }\end{array}$ \\
\hline 50 & Late AMD & $\begin{array}{l}\text { Neovascular AMD and/or } \\
\text { any geographic atrophy }\end{array}$ & $\begin{array}{l}\text { Bilateral GA, bilateral neovascular AMD, or } \\
\text { neovascular AMD in one eye and GA in the other }\end{array}$ \\
\hline
\end{tabular}

AMD, age-related macular degeneration. ${ }^{a}$ Any definite hyper- or hypopigmentary abnormalities associated with medium or large drusen but not associated with known disease entities [10].

a data package from the first cross-sectional part, and (2) a final data package from the longitudinal part of the MACUSTAR clinical study (see below) at the end of the funding period.

Terminology Clarifications

All measures developed in this project are referred to as outcome measures, in lieu of a clinical endpoint, which requires acceptance by regulatory authorities and payers.

\section{Results}

\section{Study Design}

The outcome measures will be assessed in both parts of the MACUSTAR clinical study, the cross-sectional and the longitudinal part (Fig. 1).

These parts are operationally combined under one study protocol for synergies as they include identical assessments, identical functional and imaging devices, identical clinical sites and investigators, and for iAMD a partially overlapping patient sample (Fig. 1).

The two parts of the study are:

(1) Cross-sectional part. It consists of a comparative analysis of functional, structural, and patient-reported outcomes in age-matched subjects with normal ocular aging changes only (no AMD), and in patients with early AMD, iAMD, and late-stage AMD. For this part, two visits will be analyzed; the baseline visit (day 1) and a shortterm follow-up visit ( $21 \pm 7$ days). The total number of patients in the cross-sectional part is 300 . The data generated will be used for a technical evaluation including reli- ability of tests as well as ability to discriminate between the different AMD stages and controls.

(2) Longitudinal part. A 3-year longitudinal followup of patients with iAMD to observe change in candidate endpoints over time and especially with progression from iAMD to late-stage AMD (GA or nAMD). The longitudinal part will comprise all iAMD patients from the crosssectional part $(n=150)$ and additional 450 iAMD patients as well as the 50 early AMD patients (total $n=650$ ). The data generated will be used to assess the predictive power of endpoint candidates for progression of vision impairment in $\mathrm{AAMD}$ and progression to late AMD.

Inclusion and allocation to study groups will be based on the disease stage of both eyes (Table 1). Late-stage AMD will be defined as follows: GA will be defined as a retinal area with a severely reduced signal by fundus autofluorescence and with a minimum area size of $0.1 \mathrm{~mm}^{2}$ as quantified by RegionFinder software, correlating to loss of the outer nuclear layer and the occurrence of choroidal signal enhancement on spectral-domain optical coherence tomography (SD-OCT) and spatially confined to either complete or incomplete depigmentation or other AMD typical changes (e.g., hyperpigmentation, crystalline deposits) on color fundus photography (CFP), provided any hemorrhages or exudates are absent. Finally, no leakage must be present on fluorescence angiogram (FA) (examination at the discretion of the investigator). $\mathrm{CNV}$ will be defined based on CFP, SD-OCT, and/or FA (optional). Evidence of $\mathrm{CNV}$ must include at least 2 of the following within a radius of $3,000 \mu \mathrm{m}$ of the fovea: (1) se-
64

Ophthalmologica 2019;241:61-72

DOI: $10.1159 / 000491402$
Finger et al. 
Table 2. Inclusion and exclusion criteria for the MACUSTAR clinical study

\begin{tabular}{|c|c|c|}
\hline $\begin{array}{l}\text { Inclusion criteria } \\
\text { General }\end{array}$ & $\begin{array}{l}1 \\
2 \\
3\end{array}$ & $\begin{array}{l}\text { Male and female subjects } \\
\text { Aged 55-85 years at baseline } \\
\text { Able and willing to provide written informed consent and to comply with the study protocol visits and } \\
\text { assessments; in case the subject is physically incapable of signing the ICF, this can be provided by a legally } \\
\text { acceptable representative of the subject or impartial witness }\end{array}$ \\
\hline No AMD & 1 & No signs of early, intermediate, or late AMD in both eyes \\
\hline Intermediate AMD & $\begin{array}{l}1 \\
2\end{array}$ & $\begin{array}{l}\text { Study eye must have iAMD and } \\
\text { The fellow eye must have iAMD and/or, in addition, extrafoveal GA (no atrophy within the central } \\
\text { ETDRS subfield), maximum total GA size is } 1.25 \mathrm{~mm}^{2} \\
\text { Definition of iAMD: large drusen }>125 \mu \mathrm{m} \text { and/or any AMD pigmentary abnormalities that are definite } \\
\text { hyper- or hypopigmentary abnormalities associated with medium or large drusen but not associated with } \\
\text { other known disease entities; if both eyes are eligible for the study based on inclusion criteria, the eye with } \\
\text { better visual acuity (i.e., on BCVA) will be selected as the study eye as this will improve reliability of } \\
\text { functional testing and quality of image acquisition and thus improve sensitivity to detect change over } \\
\text { time; In cases in which both eyes have the same visual acuity, the study eye will be selected at random by } \\
\text { the investigator } \\
\text { ETDRS letter chart BCVA in the study eye not worse than } 72 \text { letters (approximately } 20 / 40 \text { Snellen VA } \\
\text { equivalent) }\end{array}$ \\
\hline Late AMD & & $\begin{array}{l}\text { Subjects with bilateral GA, bilateral nAMD or nAMD in one eye and GA in the other } \\
\text { BCVA between 20/80 and 20/200 in study eye }\end{array}$ \\
\hline $\begin{array}{l}\text { Exclusion criteria } \\
\text { General }\end{array}$ & $\begin{array}{l}5 \\
6 \\
7 \\
8 \\
9\end{array}$ & $\begin{array}{l}\text { Media opacity or eye movement disorder (nystagmus) that interferes with retinal imaging data quality in } \\
\text { the opinion of the investigator } \\
\text { Severe ptosis, extraocular motility restriction or head tremor preventing adequate fundus visualization in } \\
\text { the opinion of the investigator } \\
\text { Any signs of nAMD or GA (does not apply to the late AMD group) } \\
\text { Any concurrent intraocular condition in the study eye (e.g., glaucoma or cataract) that, in the opinion of } \\
\text { the investigator would either require surgical intervention during the study to prevent or treat visual loss } \\
\text { that might result from that condition or affect interpretation of study results } \\
\text { Severe nonproliferative diabetic retinopathy, or proliferative diabetic retinopathy } \\
\text { Any diabetic macular edema or macular disease } \\
\text { Ocular disorders in the study eye (i.e., preretinal membrane) at the time of enrolment that may confound } \\
\text { interpretation of study results and compromise visual acuity } \\
\text { Diagnosis of uncontrolled glaucoma with intraocular pressure of }>30 \text { mm Hg (despite current } \\
\text { pharmacological or nonpharmacological treatment) } \\
\text { Known systemic illness which in the opinion of the investigator will prevent from actively participating in } \\
\text { the study } \\
\text { Concomitant treatment for AMD in either eye (concomitant use of vitamins/supplements is not excluded; } \\
\text { does not apply to the late AMD group) } \\
\text { Any periocular or intravitreal injections (IVT) in either eye (does not apply to the late AMD group) } \\
\text { Participation in any other interventional trial } \\
\text { Obvious retinal changes due to causes other than AMD (e.g., evidenced by an existing diagnosis of } \\
\text { monogenetic macular dystrophies, Stargardt disease, cone rod dystrophy, or toxic maculopathies) } \\
\text { Any history of allergies to fluorescein } \\
\text { Cognitive impaired subjects, illiterate, and subjects who do not speak the national language }\end{array}$ \\
\hline
\end{tabular}

\begin{tabular}{lll}
\hline No AMD & 1 & Early to late AMD (following Beckman classification) in any eye \\
\hline Early AMD & 1 & Intermediate or late AMD (following Beckman classification) in any eye \\
\hline Intermediate AMD & 1 & Any GA in the study eye \\
& 2 & Any extrafoveal GA larger than $1.25 \mathrm{~mm}^{2}$ (as defined above) in the fellow eye \\
\hline
\end{tabular}

Late AMD (All general exclusion criteria only) 
rous detachment of the sensory retina; (2) subretinal/ retinal hemorrhage; (3) pigment epithelial detachment (excluding drusenoid pigment epithelial detachment); (4) fibrous tissue; (5) hard exudates; (6) disciform scar. Through the use of multimodal functional testing and imaging, subclinical stages of late AMD are likely to be identified during the study. As the MACUSTAR study (amongst others) aims at finding early markers of disease progression in patients with iAMD, results indicating possible progression will not replace the diagnostic criteria of late-stage AMD described above.

Note that the Beckman classification of AMD defines disease stage per the individual eye. In MACUSTAR, the same disease stage in both eyes of one subject is generally required. Two exceptions have been defined in the study protocol. First, small GA areas up to a maximum lesion size of $1.25 \mathrm{~mm}^{2}$ may be present in the fellow eye for the iAMD group, provided extrafoveal location is present. Second, no distinction between GA and neovascular AMD is applied in the late AMD group.

For each patient, one eye is selected as study eye. If both eyes are eligible for the study based on inclusion criteria (Table 2), the eye with better BCVA will be selected as the study eye. In cases in which both eyes have the same visual acuity, the study eye will be selected by the investigator. Before enrollment, morphological inclusion and exclusion criteria need to be confirmed by the central reading center.

As the examinations per visit are comprehensive, patient acceptance was assessed accurately beforehand. The complete visit duration was 3 to $4 \mathrm{~h}$, which was not reported to be inacceptable by any patients.

The MACUSTAR clinical study will be carried out in 20 clinical sites (see online suppl. material) of the European Vision Clinical Research Network (EVICR.net), a dedicated network for multinational ophthalmology clinical research. These clinical sites will identify subjects with AMD as well as controls mainly from regular ophthalmologic consultations, inform them about the study and screen those subjects interested in participating.

\section{Sample Size}

The cross-sectional part of the MACUSTAR clinical study will recruit 50 normal, 50 early AMD, 50 late AMD, and 150 iAMD patients across the three age categories 5564 years, $65-74$ years, and $\geq 75$ years (age limit 85 years at baseline). Although limited information is available on test-retest reliability and on the distributions of the outcome measures within the four disease stages (no disease, early, intermediate, and late AMD), these numbers are justified by the following considerations [13]: (a) If one considers a continuous outcome measure and assumes a moderately high effect size (Cohen's $f=0.34$ ) in one-way analysis of variance, 50 patients per disease stage will be sufficient to detect mean differences between the four groups with $80 \%$ power at the $5 \%$ alpha level of significance (overall $F$-test). In addition, the planned sample size will be sufficient to detect differences with $80 \%$ power at the Bonferroni-Holm-corrected 5\% alpha level in post hoc tests comparing the mean outcome in the iAMD group to the respective means in the other patient groups. (b) The analysis of the test-retest reliability of continuous outcomes in the iAMD patient group will be based on intraclass correlation coefficients (ICCs). Assuming that there are two ratings per patient and that the minimum acceptable ICC is 0.75 , based on available data for microperimetry, 150 iAMD patients will be sufficient to reject the null hypothesis "ICC $\leq 0.75$ " for true values of ICC $>0.85$ with $80 \%$ power at the one-sided $2.5 \%$ alpha level $[14,15]$.

For the longitudinal part of the MACUSTAR clinical study, a total of 600 iAMD patients across the age categories mentioned above will be recruited and followed up 6 -monthly over 3 years. Although currently available data on the change in structural, functional or patient-related outcome measures preceding progression from iAMD to late AMD are insufficient to accurately calculate a sample size, this number is justified by the following considerations: using available data on microperimetric changes in retinal sensitivity [10], which is one of the key candidate methodologies for functional biomarkers in iAMD, and assuming constant rates of change $(\mathrm{dB} / \mathrm{y})$, the average 3-year longitudinal changes in microperimetry were estimated to be $-1.26 \mathrm{~dB}$ in eyes that show morphological AMD progression in terms of changes in drusen or pigmentation according to two experienced graders and $-0.93 \mathrm{~dB}$ in eyes that do not. Due to a lack of data in the literature correlating pointwise sensitivity from microperimetry with progression of $A M D$, the outlined reference group was chosen as a substitute. The subjects progressing morphologically are assumed to have a noticeably increased risk for progression of the disease to late AMD. Based on data from a recent iAMD cohort that has been followed up for 3 years [16], approximately 23\% of iAMD patients are expected to progress to late AMD within the 3 -year study period. Assuming normally distributed change rates and a dropout rate of $20 \%$ after 3 years, an estimated number of $n=600$ patients will be sufficient to detect a $[(-1.26)-(-0.93)] \mathrm{dB}=-0.33 \mathrm{~dB}$ difference in microperimetry change rates between possible progressors and nonprogressors (alpha $=5 \%)$. This estimation is
66

Ophthalmologica 2019;241:61-72 DOI: $10.1159 / 000491402$
Finger et al. 
based upon the very limited longitudinal data regarding mesopic microperimetry in individuals with AMD. At the time the study was planned, no relevant studies on scotopic microperimetry in AMD were available. Power will be $>80 \%$ if the standard deviations of the change rates are smaller than the absolute values of the mean change rates (i.e., $\mid$ mean $/ \mathrm{SD} \mid>1$, an assumption justified by the results by $\mathrm{Wu}$ et al. [10]). The sample size of 600 iAMD patients will be sufficient to develop a prediction model for progression to late AMD (combining 3-year changes in microperimetry, dark adaption, low luminance acuity [LLA] and other measures) and to estimate true- and false-positive rates at a high precision. For example, since the expected number of late AMD events will be approximately equal to 110 (assuming a dropout rate of $20 \%$ after 3 years and a progression rate of $23 \%$ ), there will be approximately 25 events per variable in a four-variable prediction model in tenfold cross-validation and more than 20 events per variable in fivefold cross-validation [17]. Furthermore, assuming that the sensitivity and specificity rates of the prediction model range between 95 and $99 \%$, a sample size of $n=600$ patients will result in $95 \%$ confidence intervals for tenfold-cross-validated sensitivities and specificities that are narrower than 0.11 and that have lower bounds above 0.89 [18].

To avoid low data quality and missing data, the electronic case report form has built in data checks, avoids ambiguity, and has a user-friendly design. The central reading center, one of the project partners, the Association for Innovation and Biomedical Research on Light and Image (AIBILI), and clinical data monitors will perform ongoing data quality checks.

\section{Outcome Measures}

Based on available literature and expert consensus as outlined above, the following outcomes measures will be integrated as candidates for clinical validation in iAMD into the MACUSTAR clinical study, a natural history study in iAMD for validation of clinical endpoints.

\section{Functional Outcome Measures}

Patients with iAMD frequently report difficulties in dim illumination (e.g., reading menus in restaurants) and in low contrast situations (e.g., navigating staircases, driving at night, driving through tunnels, etc.) $[11,19,20]$. Against this background, a set of functional vision tests conducted at low luminance will be validated to reliably cover the functional impairment in iAMD. Also, the course of these functional outcomes during progression to late stage AMD will be assessed.

MACUSTAR - Clinical Validation of iAMD Endpoints
For all functional tests, we will assess functional changes meaningful to the participant - referred to as minimally important difference (MID). Combining functional test results with patient-related outcome (PRO) assessments will allow us to capture MIDs for all functional tests by systematically assessing whether a clinically assessed change in function translates to a patient-reported change in functioning, as well as patient preferences (i.e., utility).

The following tests of visual function will be performed in both parts of the MACUSTAR clinical study (crosssectional and longitudinal) in addition to standard highcontrast BCVA.

(1) Fundus-controlled perimetry (microperimetry). Rubin and coworkers [21] developed the first scotopic microperimeter by adding neutral density filters to a commercial photopic instrument (Nidek MP1, Nidek Technologies, Italy) to reduce the luminance of background and test stimuli. In a recent, exploratory study of 20 patients with advanced AMD, they found scotopic microperimetry to be the best predictor of patient-reported difficulties under low light as measured by the Low Luminance Questionnaire (LLQ). In patients with iAMD, localized scotopic dysfunction is more pronounced compared to mesopic dysfunction in areas with reticular drusen, a risk factor for AMD progression [22]. Preparatory assessments showed that where early scotopic microperimeters had problems with testing accuracy, speed, and acceptability to patients, the scotopic MAIA (S-MAIA, Centervue, Italy) is easier to operate, with less operator-induced variability. Further, it appears to be less affected by ceiling and floor effects due to its extended range for both mesopic and scotopic testing $[23,24]$. Therefore, the MACUSTAR consortium will use the S-MAIA microperimeter for this project. As persons with AMD are known to adapt to reduced light levels more slowly than people without the condition [25], preliminary data are currently collected to determine the optimal duration of dark adaptation for the mesopic and scotopic tests in this patient cohort. The aim is to ensure that all participants are at a steady state level of adaptation when the microperimetry tests are conducted.

(2) LLA. The Salisbury Eye Evaluation (SEE) is a simple test of vision at low luminance [26]. All that was required was a pair of dark sunshades which reduce luminance by $1.4 \log$ units, i.e. a factor of 25 . This moves the test into the mesopic range. Using a neutral density filter that reduced the luminance by 2 log units, Sunness et al. [27] showed that an LLA deficit at baseline was a good predictor of GA progression. Finger and colleagues [11] found that the LLA deficit predicts patient-reported difficulties with night vision in iAMD.

Ophthalmologica 2019;241:61-72 
(3) Moorfields Acuity Test (MAT; Vanishing Optotypes). Shah et al. [28] have developed a new set of optotypes for measuring visual acuity that consist of pseudo high-pass letters. The authors have demonstrated that the new optotypes have better test-retest variability than traditional optotypes, and recent evidence suggests that they are more sensitive to vision loss in early AMD to iAMD [29].

(4) Dark adaptation. Impaired dark adaptation in AMD patients results in patient-reported difficulties with night vision, especially when moving from high to low ambient illumination [25]. In a study of 325 normal participants, abnormal dark adaptation at baseline was a predictor of AMD onset over 3 years [30]. Longitudinal data from a small number of eyes with different stages of AMD also suggest that disease progression is reflected in advancing delays in rod-mediated adaptation [9]. Traditional dark adaptation measurement is very lengthy as it is measured until absolute threshold is reached. A faster alternative is to monitor dark adaptation only until it reaches a criterion threshold. A recently developed and introduced commercial instrument, the AdaptDx (Maculogix, USA), will be used in the MACUSTAR clinical study to measure the so-called rod intercept time (time to reach a threshold located within the second component of rod recovery). Adaptation is assessed for up to $20 \mathrm{~min}$ at a single location just outside the macula [31]. The standard diagnostic protocol for the device is based on a pass/fail criterion, where an adaptation time falling outside of a specific window is considered to be "abnormal" [31]. For a longitudinal study, this is an insufficiently precise outcome. Thus, we will determine the optimal bleach intensity and retinal location for testing people with iAMD. The aim of this standardization process is to select testing parameters, which will allow an actual value for rod intercept time to be obtained for the majority of participants with iAMD within a 20 -min recording period. In addition, the AdaptDx will be used to measure Absolute Rod Threshold, the minimum intensity of light detectable when fully dark adapted.

(5) Contrast sensitivity (CS). A number of small studies have evaluated the effect of AMD on CS to various spatial frequencies, and evidence suggests that a mid-high spatial frequency deficit is present [32]. However, there is a paucity of data evaluating the ability of CS to predict and monitor disease progression in individuals with iAMD. Given the strong relationship between reduced CS and self-reported problems performing activities of daily living [33], an assessment of CS has the potential to provide a clinically meaningful outcome measure, which is why a classic chart-based test of CS (Pelli Robson) will be included.

(6) Performance-based tests (PBTs): Reading Speed and Navigation Performance. PBTs are standardized tests of everyday activities. In MACUSTAR, we will use Reading Speed and Navigation Performance, both performed under low illumination, to help validate the outcome of functional assessments and PROs against activities of daily life (ADL). For the reading PBT, we will use the International Reading Speed Texts (IReST) developed by members of this consortium, translated and standardized for all major European languages [34].

Navigational PBTs will utilize two unique facilities the Pedestrian Accessibility and Mobility Laboratory (PAMELA) in London and Streetscape in Paris, Fondation Voir et Entendre - FVE. Both facilities allow standardized illumination levels that can simulate walking through a residential street scene at night and are the only such facilities available in Europe. Conditions are comparable at both facilities, i.e. the same levels of illumination, etc. Navigational performance will be assessed in the cross-sectional part of the study.

\section{Structural Outcomes Measures}

To date, the state-of-the-art in retinal imaging of nonneovascular, atrophic late AMD progression is based on multimodal retinal imaging which combines several imaging approaches. These include:

- CFP

- SD-OCT more recently supplemented by OCT-angiography

- Blue and green confocal scanning laser ophthalmoscopy (cSLO) fundus autofluorescence

- Near-infrared cSLO reflectance imaging.

Image data acquisition will be carried out according to standard operational procedures, including electronic data transmission to the reading center and certification of study-site technicians and photographers. Trained and independent readers will independently and systematically analyze imaging data according to predefined grading parameter. This also includes final confirmation of development of late AMD in any eye during the study (for specific criteria see Table 1 ).

A large number of previous and current clinical studies assessing AMD progression still rely on CFP. However, this is not sufficiently sensitive or specific for structural assessment of the complex retinal abnormalities in iAMD. An optimized combination of these existing imaging technologies has not been determined and clini-
68

Ophthalmologica 2019;241:61-72 DOI: $10.1159 / 000491402$
Finger et al. 
cally validated but will be the basis for an in-depth assessment of structural abnormalities and their progression in iAMD.

As the latest technological advancements provide continuously growing number of imaging modalities, retinal imaging produces an increasing amount of data. These data provide unique high-resolution structural disease information and allow us to potentially measure an array of relevant imaging biomarkers. However, the current state-of-the-art in retinal imaging analysis uses visual inspection to extract these biomarkers. With stringent application of multimodal imaging approaches, there is a large volume of multimodal images to be analyzed. $\mathrm{Hu}$ mans are unable to efficiently exploit all the acquired information, and commonly only a limited number of imaging biomarkers are extracted. Moreover, accuracy and reliability of measurements vary within and between graders, leading to imprecise biomarker assessment and reduced prognostic power.

Automatic and semiautomatic computerized quantification of imaging biomarkers could substantially add to efficient exploitation of multimodal image information, obtaining faster, more accurate, and more reproducible measurements. Developed algorithms will be implemented in the central reading center for a validation against the current gold standard of manual grading. Currently available algorithms have several limitations. The most commonly available algorithms measure the thickness of the retina or sublayers within the retina, but these may be inaccurate in the presence of disease [35], and are not well correlated to functional deficits. Sanchez et al. [36] have been using deep learning approaches to improve the robustness of layer segmentations, which may help with both problems. Other features available commercially include measures of elevation of the RPE, which may be correlated to likelihood of progression [35]. It will be useful to see if these are also correlated to functional deficits. There are numerous other morphological features that are known to be associated with severity of disease or risk of progression such as reticular pseudodrusen, but these are not yet quantified by any commercial software. Sánchez et al. [36] and others [37-41] have previously designed computer-based algorithms for the automatic extraction of several morphological biomarkers based on one or several imaging modalities. Although promising, the performance of these algorithms needs to be further improved to detect small, characteristic changes in iAMD that can be correlated to function, patient reports, or risk of progression if they were to be utilized in future CTs. Also, the functional relevance of any of the structural can- didate biomarkers needs to be validated against functional changes to support their clinical relevance for future clinical endpoints.

\section{Patient-Reported Outcome Measures}

As highlighted above, persons with iAMD often report difficulties with contrast and low luminance. We found that self-reported night vision symptoms are associated with low luminance deficit [11] and the degree of selfreported problems with night vision could be shown to be predictive of disease progression from early to late AMD (especially GA) as well as a loss of BCVA $\geq 3$ lines over a period of 6 years $[42,43]$.

However, most currently available vision-related PRO measures (PROMs) are focused on assessing the impact of visual loss, and almost always have a ceiling effect (i.e., patients report no problems or tasks are too easy) in persons with good BCVA as is the case in iAMD. Thus, they are poorly targeted to the study population, and better targeted PROMs need to be developed. While there are instruments that measure different aspects of visual functioning and vision-related quality of life including subjective dysfunction in low luminance (e.g., Low Luminance Questionnaire, NEI Visual Functioning Questionnaire 25 ), no vision-related $P R O$ has been accepted by regulatory authorities as a clinical endpoint since recent guidelines for development of such tools have not been systematically followed. To allow drug development, which will target the low-luminance and low-contrast visual impairment of patients with iAMD, a novel PRO needs to be developed in accordance with existing guidelines from regulatory authorities. Results from this new PRO will also need to be correlated with functional endpoints, and PBT to understand the relationship between measures of function and patient report.

In order to allow for economic assessments of vision treatments alongside iAMD CTs, a utility measure sensitive to changes in vision is needed. Conventional generic utility measures such as the EQ-5D have been shown to be inappropriate in vision [44]. The added value of a number of recent developments in the field including the EQ-5D vision "bolt-on" that provides an extra vision dimension [45] and Vision Function Questionnaire Utility Index (VFQ-UI) [46] remains unclear. Thus, in order to allow for optimal utility capture in iAMD CTs a health state utility index will be developed to allow utility values to be generated alongside the collection of PRO data to ensure that future CTs generate data on patient-relevant benefits that can be utilized in cost-effectiveness analyses by HTA and reimbursement authorities. 


\section{Statistical Analysis}

Since the aim of the cross-sectional part of the clinical study is to evaluate the sensitivity, repeatability, and discriminant ability of the candidate endpoints described above, statistical analysis of these endpoints will be based on methods for measuring agreement and for analyzing differences between the disease groups. This will be done by computing intraclass correlation coefficients (ICCs) with $95 \%$ confidence intervals for continuous outcomes and Fleiss' kappa coefficients with 95\% confidence intervals for ordinal outcomes. Graphical analysis of agreement will be performed using scatterplots and Bland-Altman plots for continuous outcomes, and heat maps for ordinal outcomes.

In the longitudinal part of the study, multivariable regression and discrete time-to-event analyses will be conducted in order to assess the associations of changes in functional, structural and PRO measures with progression to late AMD. Both, progressions to $\mathrm{nAMD}$ and to GA will be considered as progression events. Based on these exploratory models, we will develop a predictive model using the rate of change in one or several functional measures, structural measures and/or PRO measures to predict progression to late-stage AMD. For this analysis, rates of change over time will be established for all functional, structural, and PRO measures, and "points of no return" will be assessed for structural measures. This will be done for (a) all progression events to latestage AMD and for (b) nAMD and GA separately. Results will be compared to identify biomarkers suggested to be specific for progression to one or the other latestage AMD type. For predictive modelling, we will consider logistic regression, but will also investigate the use of statistical learning methods such as random forests, artificial neural networks, and support vector machines. Predictive models will be derived and evaluated by repeated k-fold cross-validation [47]. Accuracy of the models will be evaluated on the test datasets using performance measures such as AUC and partial AUC [47]. Structural-functional correlations such as the correlation of localized retinal changes to quantitative results of fundus-controlled perimetry will be performed. Reporting of the various prediction models will follow the principles and guidelines of the TRIPOD Statement [48].

A combination of functional information from microperimetry measurements and structural information from multimodal retinal imaging may further improve the performance of these two individual biomarkers. These kinds of analyses have not been performed before in patients with iAMD. It is likely that clinically significant deterioration in functional measures may be partially masked by measurement imprecision resulting in limitation in the ability to identify disease progression by the functional measurement alone. In this situation, information on novel or progressive structural deficits from multimodal retinal imaging could improve the performance of the biomarkers. Analysis of structural parameter outcomes in the same model as outcome of the functional measures would mitigate measurement variability and increase the signal-to-noise ratio. An improved signal-to-noise ratio would allow disease deterioration to be identified, therefore increasing the power of a CT to identify treatment effects. Modelling of joint outcomes has shown potential in other diseases [49-51].

Simply put, each person would have a functional "chance" and a structural "chance" of changing; then, we combine these probabilities in a Bayesian framework. Crabb and colleagues [52] have successfully done this in people with ocular hypertension, when trying to predict progression to glaucoma, using measures from standard automated perimetry and neuroretinal rim area imaging. This particular prescription simply relies on combinations of single summary measures on rate of change from the two measurement modalities, combining measurements in "higher dimensions" retaining "layers" of information from each measurement - pixel/voxel intensity in images and single visual field points [53]. The same could easily be applied to regional changes from microperimetry and specific areas of change on imaging, taking advantage of modern statistical feature extraction methods [54].

\section{Conclusions}

Herein, we outline the protocol development of the MACUSTAR clinical study which will establish a toolbox of functional, structural, and patient-reported outcome measures for use in future iAMD CTs. Likely a combination of multiple outcome measures will be used to assess progression and may serve as a surrogate endpoint for progression to late AMD. As the functional deficit in iAMD is most pronounced under low luminance and low contrast conditions, this is what we will explore in MACUSTAR.

Strengths of our protocol development are integration of available data, comprehensive expertise including biostatisticians, as well as feedback from all relevant regulators and HTA bodies. Limitations are the lack of pilot data
Finger et al. 
for the overall protocol at this stage, a possible selection bias (such as sampling bias due to the nonrandom sample out of subjects with iAMD, attrition bias), as well as the uncertainty around the sample size planning given the lack of necessary data around functional changes preceding progression from iAMD to late AMD. Considering this, our current sample size assessments are the best possible to date.

In conclusion, novel outcome measures are needed for iAMD CTs as conventional high-luminance, high-contrast functional testing, or existing PROMs are unlikely to detect functional loss or patient-relevant impact of the disease and thus cannot be used in future CTs for iAMD. A combination of candidate outcome measures assessing function under low-luminance and low-contrast conditions with structural outcomes which allow for a topographical correlation may improve precision of the measurement and should be explored.

\section{Acknowledgments}

We are very grateful for the continuous support provided by Nathalie Seigneuret at the IMI Office in Brussels. We also gratefully acknowledge the work put into this project by consortium members who have since moved on to work elsewhere.

\section{Disclosure Statement}

R.P. Finger: C Novartis, Bayer, Santen, Opthea, Novelion, RetinaImplant, Oxford Innovation; F Novartis.

S. Schmitz-Valckenberg: Alcon/Novartis C, F; Allergan F; Bayer F, R; Carl Zeiss Meditec F, R; Bioeq/Formycon F; Centervue F; Heidelberg Engineering F, R; Optos F; Genentech/Roche C, F, R.

F.G. Holz: C Acucela, Aoellis, Bayer, Boehringer-Ingelheim, Genentech/Roche, Heidelberg Engineering, Ionis, Novartis, Optos, Thea, Thrombogenics, Zeiss, F; Heidelberg Engineering, Zeiss; R Centervue, Heidelberg Engineering, Genentech/Roche, Nieghtstar.

D.P. Crabb: Allergan R; Roche R; Santen R; Centervue C.

J. Cunha-Vaz reports grants from Carl Zeiss Meditec, outside the submitted work and is consultant for Alimera Sciences, Allergan, Bayer, Gene Signal, Novartis, Pfizer, Precision Ocular Ltd., Roche, Sanofi-Aventis, Vifor Pharma, and Carl Zeiss Meditec.

F. Asmus is an employee of Bayer Pharma AG, Berlin, Germany. M. Durbin is an employee of Carl Zeiss Meditec AG.

U.F.O. Luhmann is an employee of F. Hoffmann-La Roche Ltd.

P. Margaron, G. Normand, and Parisa Zamiri are employees of Novartis.

\section{Funding Sources}

This project has received funding from the Innovative Medicines Initiative 2 Joint Undertaking under grant agreement No. 116076. This Joint Undertaking receives support from the European Union's Horizon 2020 research and innovation program and EFPIA and Carl Zeiss Meditec AG.

\section{References}

1 Lim LS, Mitchell P, Seddon JM, Holz FG, Wong TY. Age-related macular degeneration. Lancet. 2012 May;379(9827):1728-38.

2 Augood CA, Vingerling JR, de Jong PT, Chakravarthy U, Seland J, Soubrane G et al. Prevalence of age-related maculopathy in older Europeans: the European Eye Study (EUREYE). Arch Ophthalmol. 2006 Apr; 124(4):529-35.

3 Finger RP, Fimmers R, Holz FG, Scholl HP. Prevalence and causes of registered blindness in the largest federal state of Germany. $\mathrm{Br} J$ Ophthalmol. 2011 Aug;95(8):1061-7.

4 Colijn JM, Buitendijk GH, Prokofyeva E, Alves D, Cachulo ML, Khawaja AP et al.; EYE-RISK consortium; European Eye Epidemiology (E3) consortium. Prevalence of Age-Related Macular Degeneration in Europe: The Past and the Future. Ophthalmology. 2017 Dec;124(12): 1753-63.

5 Cruess AF, Zlateva G, Xu X, Soubrane G, Pauleikhoff D, Lotery A et al. Economic burden of bilateral neovascular age-related macular degeneration: multi-country observational study. Pharmacoeconomics. 2008;26(1):5773.

6 Holz FG, Strauss EC, Schmitz-Valckenberg S, van Lookeren Campagne M. Geographic atro- phy: clinical features and potential therapeutic approaches. Ophthalmology. 2014 May; 121(5):1079-91.

7 Rosenfeld PJ. Preventing the Growth of Geographic Atrophy: An Important Therapeutic Target in Age-Related Macular Degeneration. Ophthalmology. 2018 Jun;125(6):794-5.

8 Doody RS, Farlow M, Aisen PS; Alzheimer's Disease Cooperative Study Data Analysis and Publication Committee. Phase 3 trials of solanezumab and bapineuzumab for Alzheimer's disease. N Engl J Med. 2014 Apr;370(15):1460.

9 Jackson GR, Clark ME, Scott IU, Walter LE, Quillen DA, Brigell MG. Twelve-month natural history of dark adaptation in patients with AMD. Optom Vis Sci. 2014 Aug;91(8):925-31.

$10 \mathrm{Wu}$ Z, Ayton LN, Luu CD, Guymer RH. Longitudinal changes in microperimetry and low luminance visual acuity in age-related macular degeneration. JAMA Ophthalmol. 2015 Apr; 133(4):442-8

$11 \mathrm{Wu}$ Z, Guymer RH, Finger RP. Low luminance deficit and night vision symptoms in intermediate age-related macular degeneration. Br J Ophthalmol. 2016 Mar;100(3):395-8.

12 Ferris FL 3rd, Wilkinson CP, Bird A, Chakravarthy U, Chew E, Csaky K et al.; Beckman Initiative for Macular Research Classification
Committee. Clinical classification of age-related macular degeneration. Ophthalmology. 2013 Apr;120(4):844-51.

13 Zou GY. Sample size formulas for estimating intraclass correlation coefficients with precision and assurance. Stat Med. 2012 Dec;31(29): 3972-81.

14 Midena E, Vujosevic S, Cavarzeran F: Normal values for fundus perimetry with the microperimeter MP1. Ophthalmology 2010; 117:15716, 1576 el. https://doi.org/10.1016/j.ophtha. 2009.12.044.

15 Weingessel B, Sacu S, Vécsei-Marlovits PV, Weingessel A, Richter-Mueksch S, SchmidtErfurth U. Interexaminer and intraexaminer reliability of the microperimeter MP-1. Eye (Lond). 2009 May;23(5):1052-8.

16 Steinberg JS, Göbel AP, Thiele S, Fleckenstein M, Holz FG, Schmitz-Valckenberg S. Development of Intraretinal Cystoid Lesions in Eyes with Intermediate Age-Related Macular Degeneration. Retina. 2016 Aug;36(8):154856.

17 van der Ploeg T, Austin PC, Steyerberg EW. Modern modelling techniques are data hungry: a simulation study for predicting dichotomous endpoints. BMC Med Res Methodol. 2014 Dec;14(1):137. 
18 Nadeau C, Bengio Y. Inference for the generalization error. Mach Learn. 2003;52(3):239-81.

19 Sevilla MB, McGwin G Jr, Lad EM, Clark M, Yuan EL, Farsiu S et al. Relating Retinal Morphology and Function in Aging and Early to Intermediate Age-related Macular Degeneration Subjects. Am J Ophthalmol. 2016 May; 165:65-77.

20 Taylor DJ, Hobby AE, Binns AM, Crabb DP. How does age-related macular degeneration affect real-world visual ability and quality of life? A systematic review. BMJ Open. 2016 Dec; 6(12): 011504.

21 Crossland MD, Luong VA, Rubin GS, Fitzke FW. Retinal specific measurement of darkadapted visual function: validation of a modified microperimeter. BMC Ophthalmol. 2011 Feb;11(1):5.

22 Steinberg JS, Fitzke FW, Fimmers R, Fleckenstein M, Holz FG, Schmitz-Valckenberg S. Scotopic and Photopic Microperimetry in $\mathrm{Pa}$ tients With Reticular Drusen and Age-Related Macular Degeneration. JAMA Ophthalmol. 2015 Jun;133(6):690-7.

23 Pfau M, Lindner M, Steinberg JS, Thiele S, Brinkmann CK, Fleckenstein M, Holz FG, Schmitz-Valckenberg S: Visual field indices and patterns of visual field deficits in mesopic and dark-adapted two-colour fundus-controlled perimetry in macular diseases. $\mathrm{Br} \mathrm{J}$ Ophthalmol 2017; Published Online First: 16 November 2017: https://doi.org/10.1136/ bjophthalmol-2017-311012.

24 Steinberg JS, Saßmannshausen M, Pfau M, Fleckenstein M, Finger RP, Holz FG et al. Evaluation of Two Systems for Fundus-Controlled Scotopic and Mesopic Perimetry in Eye with Age-Related Macular Degeneration. Transl Vis Sci Technol. 2017 Jul;6(4):7.

25 Owsley C, McGwin G Jr, Jackson GR, Kallies K, Clark M. Cone- and rod-mediated dark adaptation impairment in age-related maculopathy. Ophthalmology. 2007 Sep;114(9):172835.

26 Rubin GS, West SK, Muñoz B, Bandeen-Roche $\mathrm{K}$, Zeger S, Schein O et al. A comprehensive assessment of visual impairment in a population of older Americans. The SEE Study. Salisbury Eye Evaluation Project. Invest Ophthalmol Vis Sci. 1997 Mar;38(3):557-68.

27 Sunness JS, Rubin GS, Broman A, Applegate CA, Bressler NM, Hawkins BS: Low luminance visual dysfunction as a predictor of subsequent visual acuity loss from geographic atrophy in age-related macular degeneration. Ophthalmology 2008; 115:1480-8, 1488.e1-2. https:// doi.org/10.1016/j.ophtha.2008.03.009.

28 Shah N, Dakin SC, Redmond T, Anderson RS. Vanishing Optotype acuity: repeatability and effect of the number of alternatives. Ophthalmic Physiol Opt. 2011 Jan;31(1):17-22.

29 Shah N, Dakin SC, Dobinson S, Tufail A, Egan CA, Anderson RS. Visual acuity loss in patients with age-related macular degeneration measured using a novel high-pass letter chart. Br J Ophthalmol. 2016 Oct;100(10): 1346-52.

30 Owsley C, McGwin G Jr, Clark ME, Jackson GR, Callahan MA, Kline LB et al. Delayed RodMediated Dark Adaptation Is a Functional Biomarker for Incident Early Age-Related Macular Degeneration. Ophthalmology. 2016 Feb;123(2):344-51.

31 Jackson GR, Owsley C, McGwin G Jr. Aging and dark adaptation. Vision Res. 1999 Nov; 39(23):3975-82.

32 Hogg RE, Chakravarthy U. Visual function and dysfunction in early and late age-related maculopathy. Prog Retin Eye Res. 2006 May; 25(3):249-76.

33 Haymes SA, Johnston AW, Heyes AD. Relationship between vision impairment and ability to perform activities of daily living. Oph thalmic Physiol Opt. 2002 Mar;22(2):79-91.

34 Rubin GS. Measuring reading performance. Vision Res. 2013 Sep 20;90:43-51.

35 Wintergerst MW, Schultz T, Birtel J, Schuster AK, Pfeiffer N, Schmitz-Valckenberg S et al. Algorithms for the Automated Analysis of Age-Related Macular Degeneration Biomarkers on Optical Coherence Tomography: A Systematic Review. Transl Vis Sci Technol. 2017 Jul;6(4):10.

36 Sanchez CI, Lambertus S, Bloemen B, Bax N, Venhuizen FG, Van Grinsven MJ et al. Automatic quantification of geographic atrophy in autofluorescence images of Stargardt patients. Invest Ophthalmol Vis Sci. 2015;56:5258.

37 Liefers B, Venhuizen FG, Schreur V, van Ginneken B, Hoyng C, Fauser S et al. Automatic detection of the foveal center in optical coherence tomography. Biomed Opt Express. 2017 Oct;8(11):5160-78.

38 van Grinsven MJ, Buitendijk GH, Brussee C, van Ginneken B, Hoyng CB, Theelen $T$ et al. Automatic identification of reticular pseudodrusen using multimodal retinal image analysis. Invest Ophthalmol Vis Sci. 2015 Jan;56(1): 633-9.

39 van Grinsven MJ, Lechanteur YT, van de Ven JP, van Ginneken B, Hoyng CB, Theelen T et al. Automatic drusen quantification and risk assessment of age-related macular degeneration on color fundus images. Invest Ophthalmol Vis Sci. 2013 Apr;54(4):3019-27.

40 Venhuizen FG, van Ginneken B, Liefers B, van Grinsven MJ, Fauser S, Hoyng C et al. Robust total retina thickness segmentation in optical coherence tomography images using convolutional neural networks. Biomed Opt Express. 2017 Jun;8(7):3292-316.

41 Venhuizen FG, van Ginneken B, van Asten F, van Grinsven MJ, Fauser S, Hoyng CB et al. Automated Staging of Age-Related Macular Degeneration Using Optical Coherence Tomography. Invest Ophthalmol Vis Sci. 2017 Apr;58(4):2318-28.

42 Ying GS, Maguire MG, Liu C, Antoszyk AN; Complications of Age-related Macular Degeneration Prevention Trial Research Group.
Night vision symptoms and progression of age-related macular degeneration in the Complications of Age-related Macular Degeneration Prevention Trial. Ophthalmology. 2008 Nov;115(11):1876-82.

43 Ying GS, Maguire MG; Complications of Agerelated Macular Degeneration Prevention Trial Research Group. Development of a risk score for geographic atrophy in complications of the age-related macular degeneration prevention trial. Ophthalmology. 2011 Feb; 118(2):332-8.

44 Tosh J, Brazier J, Evans P, Longworth L. A review of generic preference-based measures of health-related quality of life in visual disorders. Value Health. 2012 Jan;15(1):118-27.

45 Longworth L, Yang Y, Young T, Mulhern B, Hernández Alava M, Mukuria $C$ et al. Use of generic and condition-specific measures of health-related quality of life in NICE decisionmaking: a systematic review, statistical modelling and survey. Health Technol Assess. 2014 Feb;18(9):1-224.

46 Rentz AM, Kowalski JW, Walt JG, Hays RD, Brazier JE, Yu R et al. Development of a preference-based index from the National Eye Institute Visual Function Questionnaire-25. JAMA Ophthalmol. 2014 Mar;132(3):310-8.

$47 \mathrm{Kim} \mathrm{JH}$. Estimating classification error rate: repeated cross-validation, repeated hold-out and bootstrap. Comput Stat Data Anal. 2009; 53(11):3735-45.

48 Collins GS, Reitsma JB, Altman DG, Moons KG. Transparent Reporting of a multivariable prediction model for Individual Prognosis or Diagnosis (TRIPOD): the TRIPOD statement. Ann Intern Med. 2015 Jan;162(1):55-63.

49 Frost C, Kenward MG, Fox NC. The analysis of repeated 'direct' measures of change illustrated with an application in longitudinal imaging. Stat Med. 2004 Nov;23(21):3275-86.

50 Harvey DJ, Beckett LA, Mungas DM. Multivariate modeling of two associated cognitive outcomes in a longitudinal study. J Alzheimers Dis. 2003 Oct;5(5):357-65.

51 Schott JM, Frost C, Whitwell JL, Macmanus DG, Boyes RG, Rossor MN et al. Combining short interval MRI in Alzheimer's disease: implications for therapeutic trials. J Neurol. 2006 Sep;253(9):1147-53.

52 Russell RA, Malik R, Chauhan BC, Crabb DP Garway-Heath DF. Improved estimates of visual field progression using bayesian linear regression to integrate structural information in patients with ocular hypertension. Invest Ophthalmol Vis Sci. 2012 May;53(6):2760-9.

53 Zhu H, Crabb DP, Schlottmann PG, Lemij HG, Reus NJ, Healey PR et al. Predicting visual function from the measurements of retinal nerve fiber layer structure. Invest Ophthalmol Vis Sci. 2010 Nov;51(11):5657-66.

54 Zhu H, Poostchi A, Vernon SA, Crabb DP. Detecting abnormality in optic nerve head images using a feature extraction analysis. Biomed Opt Express. 2014 Jun;5(7):2215-30. 